\title{
Can Oral Appliances be an Alternative Treatment for Severe Obstructive Sleep Apnea Syndrome Patients?
}

\section{Aida M. Yousef ${ }^{1 *}$ and Fatma El-Waseef ${ }^{2}$}

${ }^{1}$ Lecturer of Chest Medicine, Mansoura University, Egypt

${ }^{2}$ Faculty of Dentistry, Mansoura University, Egypt

\begin{abstract}
Background: Obstructive Sleep Apnea Syndrome (OSAS) is recognized as a serious health problem that impairs quality of life. Although, optimal treatment of OSAS with continuous positive airway pressure (CPAP) reduce the number of respiratory events during sleep, and thus improve quality of life, a large number of patients do not tolerate CPAP. In the last decade, there has been an explosion of interest in the using of oral appliances to treat OSAS; however, in severe OSAS there is only scanty evidence available indicating that dental appliances are effective.
\end{abstract}

Objectives: This study aimed at evaluating the efficacy of the mandibular advancing appliance (MAA) in the treatment of severe OSAS patients.

Material and Methods: Thirty three polysomnography (PSG) diagnosed cases of severe OSAS patients were included. The mean apnea/hypopnea index (AHI) was $42.36 \pm 7.92$. The cases were subjectively assessed by the Epworth Sleepiness Scale (ESS). Outcome measurements included sleep study, sleepiness; side-effects and compliance were assessed.

Results: The polysomnographic variables and clinical assessments revealed a significant improvement in the patients.

Conclusion: Mandibular Advancement Appliance therapy can be an actual alternative treatment for severe OSAS patients who cannot tolerate CPAP. The better compliance seen with the MAA may be advantageous.

Keywords: Obstructive sleep apnea syndrome; Mandibular advancement appliance; Continuous positive airway pressure

Abbreviations: OSAS: Obstructive Sleep Apnea Syndrome; MAA: Mandibular Advancement Appliance; CPAP: Continuous Positive Airway Pressure; PSG: Polysomnography

\section{Introduction}

Obstructive Sleep Apnea Syndrome (OSAS) is a highly prevalent disease leading to severe medical consequences if left untreated [1]. OSAS affects about $2 \%-4 \%$ of the adult population. Moreover, it is the most common progressive and serious sleep breathing disorder that is responsible for more mortality and morbidity than any other sleep disorder [2]. It also impairs quality of life by increasing the risk of hypertension and cardiovascular disease [3]. Currently, it is considered to be a systemic disease [4].

Among the different treatment modalities currently available to treat OSAS is Continuous Positive Airway Pressure (CPAP) which was considered the most efficient and is known as the gold standard in the treatment of this condition. The CPAP works as a pneumatic splint to relieve airway obstruction during sleep to eliminate respiratory events [5].

However, because of physical discomfort associated with wearing CPAP, consequently, a large number of patients do not tolerate CPAP, unable to or unwilling to comply with the use of it and search for alternatives. Eventually, patient's adherence to this treatment limits its overall effectiveness [6-8].

Hence, it is considered mandatory to always seek for another comfortable, easier and effective treatment options for OSAS patients [9]. In the last decade, there has been an explosion of interest in the using of mandibular advancing appliance to treat OSAS.

Several studies concerning the effectiveness of (MAA) therapy in treating mild and moderate obstructive sleep apnea are available, however, little is known from the relevant literatures about their effectiveness and compliance with respect to severe OSAS patients $[10,11]$.

Therefore, the present work aims at evaluating efficacy of MAA therapy in severe OSAS patients.

\section{Material and Methods}

Thirty five fully dentulous or partially edentulous patients with severe degrees of OSAS were eligible for this study from Sleep Unit in the Thoracic department of our institute. The study was approved by the local ethical committee and patients gave their informed consents.

\section{Inclusion criteria}

The patients either male or female were recruited with a documented Polysomnography (PSG) diagnosed severe OSA cases (AHI $\geq 30$ events/h) and, Intolerance to CPAP use, patients who refused using CPAP, can't afford its price, patient with adequate dentition, CPAP failure, which is defined as the inability to use of CPAP $\geq 4$ hours per night on $70 \%$ of nights during a consecutive thirty (30) day period anytime during the first three (3) months of initial usage.

\section{Exclusion criteria}

Patients had previous treatment for OSAS other than the trial of

${ }^{*}$ Corresponding author: Aida M. Yousef, Lecturer of Chest Medicine, Mansoura University, Egypt, Tel: 02001020254812; E-mail: aymanhusen2002@yahoo.com

Received February 16, 2014; Accepted March 27, 2014; Published March 31, 2014

Citation: Yousef AM, El-Waseef F (2014) Can Oral Appliances be an Alternative Treatment for Severe Obstructive Sleep Apnea Syndrome Patients? J Pulm Respir Med 4: 182. doi:10.4172/2161-105X.1000182

Copyright: (c) 2014 Yousef AM, et al. This is an open-access article distributed under the terms of the Creative Commons Attribution License, which permits unrestricted use, distribution, and reproduction in any medium, provided the original author and source are credited. 
CPAP, coexisting sleep disorder other than OSAS, no significant nasal obstruction, unstable medical illness, completely edentulous cases, inadequate dental structure or temporo-mandibular joint disease contraindicating MAA treatment as assessed by the dentist.

At baseline, each patient has been assessed by PSG sleep study. Case details were recorded on a sleep disordered breathing examination form. Also Epworth Sleepiness Scale (ESS) [12] was recorded in all the cases before using the appliance. Subjects were then referred to the orthodontist for fitting an adjustable oral appliance. Lateral cephalometric radiographs were done without $\mathrm{OA}$ at baseline and with $\mathrm{OA}$ in situ at six months. On the cephalometric radiographs Overjet (OJ) and MP-H distance were measured. Overjet is the horizontal distance between upper and lower incisors with reference to the upper occlusal plane. MP-H (distance from the mandibular plane to the most anterior point of the hyoid bone), as shown in Figure 1a and 1b. All subjects underwent sleep studies with OA at 6-month.

\section{Polysomnograpgic sleep study}

Polysomnograpgic sleep study was done if the patient complains of unintentional sleep episodes during wakefulness, daytime sleepiness, on refreshing sleep, fatigue, or insomnia, patient wakes with breath holding, gasping or choking or if the bed partner reports loud snoring, breathing interruptions, or both during the patient's sleep.

In sleep-laboratory all patients had been assessed regarding their degree of OSAS by subjecting them to basic, full night formal PSG sleep study (sleep screen recorder viasys company Germany) in the supine position for definitive diagnosis. PSG was performed and scored according to standard criteria using nasal pressure cannulae and tracheal sounds (suprasternal microphone) for airflow measurement. Respiratory events were scored manually. Apnea was defined as cessation of airflow for $\geq 10 \mathrm{~s}$. Hypopnea was defined as a $30 \%$ reduction accompanied by a $4 \%$ decrease in $\mathrm{SpO} 2$ of airflow or a $50 \%$ reduction of airflow accompanied by a $3 \%$ decrease in $\mathrm{SpO} 2$. The apnea index was taken as the average number of apnea events per hour of sleep.

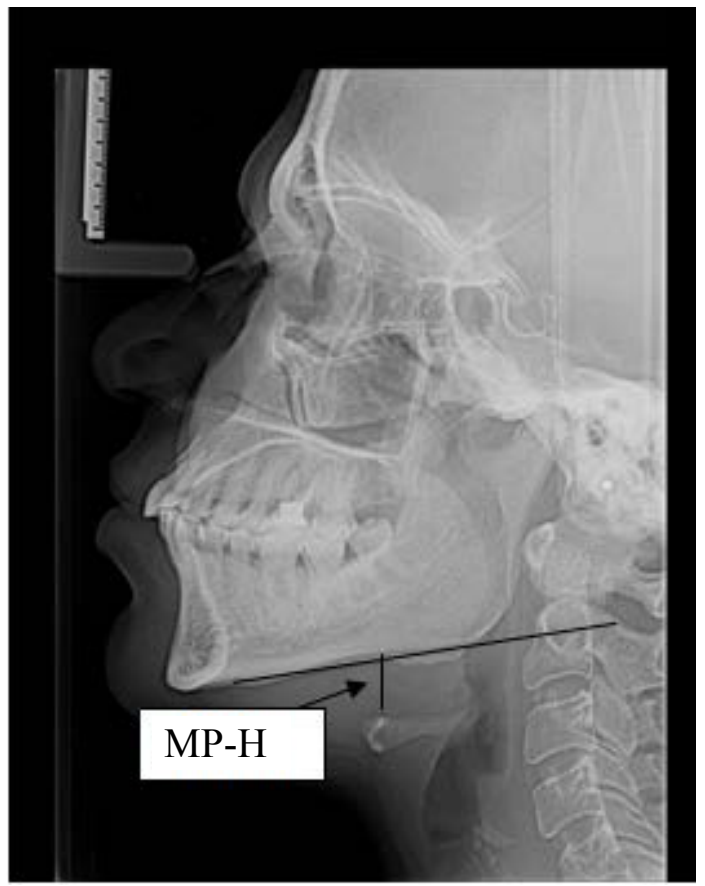

Figure 1a: Baseline cephalometry (arrow shows MP-H distance).

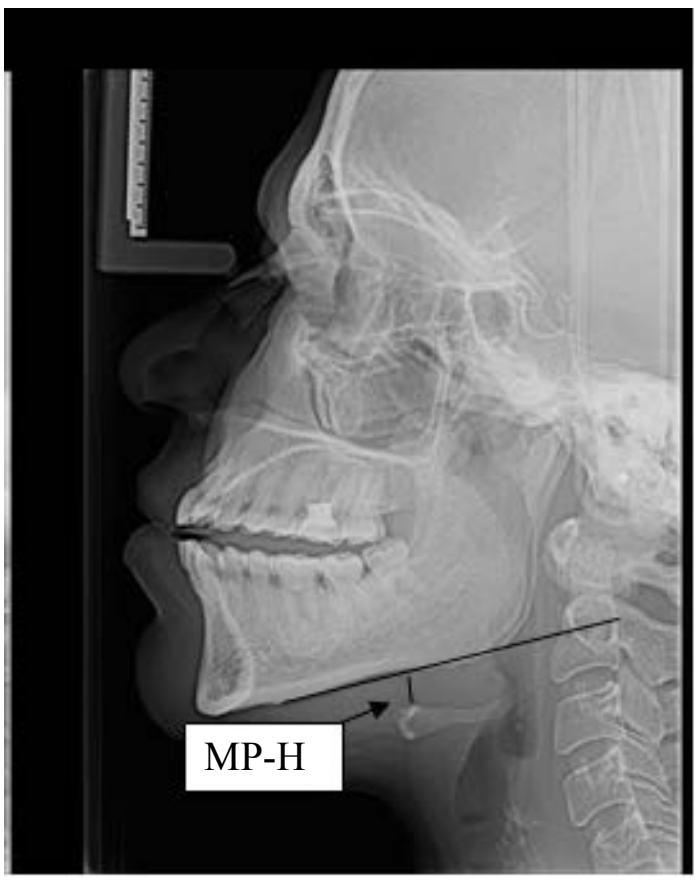

Figure 1b: Cephalometry after 6 months with MAA in situ (arrow shows MP-H distance)

The patient completed the Epworth Sleepiness Scale (ESS) [12] before and 6-month after the placement of MAA. The ESS consists of eight questions answered by the patient with regard to daily activities measured on a scale of zero to three and having proportionate values starting from a minimum of zero to a maximum of twenty-four points.

Discussion about the advantages and disadvantages of both CPAP and MAA with video helping had been made.

The total number of patients chose MAA was thirty five. They all had to agree to regular use of the oral appliance and to medical and dental follow up examination protocol.

For each patient, upper and lower primary and secondary impressions have been taken. Maxillomandibular relationship records were first obtained by fabrication of an anterior jig made from modeling compound. The patient was instructed to protrude the mandible to an edge-to-edge position with an interincisal opening of $2-3 \mathrm{~mm}$ and the selected wax recording material is used to record this relationship. Once the recording material had hardened, the jig was removed, trimmed and evaluated for fit and accuracy. The verified jig is repositioned on the teeth and posterior interocclusal records are made. The posterior records were made from a wax recording material. After mounting the casts, undercuts and large distance were blocked out with a thin mix of plaster and a small brush. The prosthesis was waxed up to a full contour in one piece, including the creation of a central vacancy for air passage. The pattern wax extended to the height of contour of the teeth are slightly engaged. The cast-pattern complex was separated from the mounting in one-piece, flasked, deflasked, finished and polished. The finished appliances were inserted into the patient's mouth to check their adaptation. The patient was instructed to wear the appliance regularly during sleep time. The appliance was inspected after one week of delivery to carefully adjust it regarding any pressure symptom.

A self-reported OA compliance and self-reported Total Sleep Time (TST) were assessed using a diary filled out by the patient on each day 
of the 6-month follow-up period during the intervals between the follow-up visits.

\section{Post-treatment response}

The clinical and PSG assessments were performed for each patient after 6 months; outcomes were assessed during the last week of the treatment period. Outcome measurements included daytime sleepiness, reported side-effects, treatment compliance, satisfaction, and the effects on sleep structure, oxygenation, and snoring index. The snoring index (SI) was defined as (total time with snoring $>90 \mathrm{~dB} /$ estimated total sleep time) $\times 100$. Compliance is considered if the mean OA use of more than 5 days per week and more than $4 \mathrm{~h}$ per day.

Complete response was considered to be a reduction in apnea/ hypopnea index (AHI) to $<5 / \mathrm{h}$. A partial response was defined as an improvement in symptoms combined with a $>50 \%$ reduction in AHI but the AHI remained $>5 / \mathrm{h}$. Treatment failure was defined as having ongoing symptoms and/or $<50 \%$ reduction in AHI. Compliance failure was defined as an inability of the patient to use OA more than 5 days per week and more than $4 \mathrm{~h}$ per day.

\section{Statistical Analysis}

The statistical analyses of data base obtained in the present study were compiled on an Excel worksheet and SPSS version 16 software program (statistical package for social science) was used for analysis. (SPSS, Chicago, IL, USA). Appropriate statistical tests of significance were carried out.

Continuous variables were described as mean \pm SD for variables with a normal distribution and median (interquartile range) for variables with a non-normal distribution. Normality of distribution was assessed using the Kolmogorov-Smirnov test. A sample size of 33 patients was calculated to detect a $1 \mathrm{SD}$ difference in AHI between pretreatment and post-treatment with a power of $99 \%$ and a significance level of 5\% (two-sided). Between pre and post treatment differences were assessed using a paired t-test and unpaired t-test for variables with a normal distribution, and by a Wilcoxon Signed-Rank test and MannWhitney test for variables with a non-normal distribution. All reported p-values are two-sided and the Bonferroni correction was used for pairwise comparisons. A p-value of 0.05 was considered to indicate statistical significance.

\section{Results}

\section{Subjects}

Two patients dropped out early in the study and were lost to follow up. The treatment results were presented on the remaining 33 patients used MAA. The demographic characteristics of the patients were shown in Table 1.

The results showed a complete response $(\leq 50 \%$ reduction in AHI and $<5$ events/h) was obtained in $36 \%$ of patients. A partial response ( $\geq 50 \%$ reduction in AHI, Post AHI remaining $\geq 5$ events/h) was observed in $45 \%$ of the cases. Treatment failure $(<50 \%$ reduction in AHI) occurred in six cases (19\%). Four out of the six cases with treatment failure had a high BMI (BMI $\geq 30)$ (Table 2).

\begin{tabular}{|l|c|c|}
\hline $\begin{array}{l}\text { Parameters } \\
\text { (Characteristic) }\end{array}$ & Mean \pm SD & Range \\
\hline AGE, years (20 males, 13 females) & $50 \pm 15$ & $35-65$ \\
\hline BMI & $29 \pm 2.5$ & $26.5-31.5$ \\
\hline ESS & $10 \pm 3$ & $7-13$ \\
\hline
\end{tabular}

Table 1: Baseline characteristic of the study patients

\begin{tabular}{|l|c|}
\hline Response & Sever $\mathbf{( N = 3 3 )}$ \\
\hline Complete Response & $(n=12) 36 \%$ \\
\hline Partial Response & $(n=15) 45 \%$ \\
\hline Treatment Failure & $(n=6) 19 \%$ \\
\hline
\end{tabular}

Table 2: Percentages of complete, partial response and treatment failure with MAA therapy.

\begin{tabular}{|l|c|c|c|}
\hline Value & Pre & Post & P \\
\hline BMI & $32 \pm 4.5$ & $32 \pm 2.9$ & $>0.05$ (NS) \\
\hline ESS & $10 \pm 3$ & $7.7 \pm 4.0$ & $<0.001$ \\
\hline
\end{tabular}

Table 3: BMI and ESS pre and post MAA.

\begin{tabular}{|l|c|c|c|}
\hline Variable & Pre & Post & P \\
\hline AHI & & & \\
Median & 42.36 & 16.66 & 0.000 \\
Minimum & 31 & 4 & \\
Maximum & 55 & 35 & \\
\hline Desaturation Index & & & \\
Median & 30.27 & 10.86 & 0.000 \\
Minimum & 20 & 1.5 & \\
Maximum & 40 & 26 & \\
\hline Lowest nocturnal desaturation (SPO2) & & & \\
Median & 81.21 & 86 & 0.000 \\
Minimum & 78 & 80 & \\
Maximum & 89 & 92 & \\
\hline
\end{tabular}

Table 4: AHI, Desaturation index and lowest nocturnal desaturation pre and post treatment.

\begin{tabular}{|l|c|c|c|}
\hline Variable & PRE & POST & P \\
\hline Sleep efficiency & $77.2 \pm 8.2 \%$ & $85.9 \pm 12 \%$ & $<0.05$ \\
\hline Stage 1-2 & $56.3 \pm 12.1 \%$ & $50.8 \pm 13.7 \%$ & $<0.05$ \\
\hline Stage 3-4 & $19.5 \pm 9.1 \%$ & $23 \pm 9.1 \%$ & $<0.05$ \\
\hline REM & $15.6 \pm 6.7 \%$ & $24.2 \pm 7.8 \%$ & $<0.001$ \\
\hline
\end{tabular}

Table 5: Show sleep stages in pre and post treatment.

No statistically significant difference in BMI $(\mathrm{p}>0.05)$ was observed between values at baseline and by 6 months after using MAA. There was a significant decrease in ESS 6 months after using MAA $(\mathrm{p}<0.001)$ (Table 3).

There were statistically significant differences $(P=0.000)$ in AHI, Desaturation index and Lowest nocturnal desaturation with MAA than pretreatment (Table 4). There was a negative correlation between MAA treatment response and AHI.

The Comparison of pretreatment data (diagnostic PSG) with post treatment data (PSG with MAA in-situ) showed a significant increase in sleep efficiency, stage 3-4 sleep (slow sleep) and stage REM sleep percent $(\mathrm{P}<0.05,<0.05$ and $<0.001$ respectively), there were significant decrease in stage 1-2 sleep percent with $\mathrm{p}<0.05$ (Table 5).

As regards to other parameters of polysomnography other than AHI and sleep stages, there were decreases in wake after sleep onset (WASO), arousal index and snoring index but increase in TST with statistically significant difference for the all Table 6 .

\section{Treatment efficacy}

At the final PSG there was a significant improvement in AHI, minimum oxygen desaturation, and total sleep time with the MAA.

\section{Symptoms and Treatment Compliance}

The common side effects of OA treatment included: TMJ discomfort $(n=8)$, mouth dryness $(n=5)$, excessive salivation $(n=4)$, teeth malalignment $(n=2)$, and toothache/ gum discomfort $(n=8)$. The 


\begin{tabular}{|l|c|c|c|}
\hline Parameters & Pre & Post & P \\
\hline SNORING & $0.87 \pm 0.1$ & $0.57 \pm 0.1$ & $<0.001$ \\
\hline TST $(m)$ & $320.3 \pm 94.1$ & $421.2 \pm 55.2$ & 0.044 \\
\hline Wake after sleep onset (WASO) $(m)$ & $32.3 \pm 21.6$ & $15.4 \pm 10.6$ & 0.018 \\
\hline ARAUSAL INDEX & $45.3(20.8)$ & $33.4(15.8)$ & $<0.001$ \\
\hline
\end{tabular}

Table 6: Comparison of other PSG parameters pre and post with MAA therapy.

\begin{tabular}{|l|c|c|c|}
\hline & Baseline & $\mathbf{6}$ months & P value \\
\hline $\mathrm{OJ}, \mathrm{mm}$ & $4.3(2.8-5.7)$ & $0.0(-0.1-0.1)($ edge to edge) & $<0.001$ \\
\hline $\mathrm{MPH}, \mathrm{mm}$ & $21.9(19.4-26.3)$ & $17.8(10.7-21.6)$ & $<0.001$ \\
\hline
\end{tabular}

Table 7: Cephalometric parameters.

average $\mathrm{OA}$ use as marked in their sleep diary was five nights per week and $6 \mathrm{~h}$ per night.

On cephalometric analyses, All subjects had some degree of retrognathism, with a median overjet (OJ) measurements of 4.3 (2.85.7) with improvement after six months with MAA (Table 7). There is an increase of vertical opening in average $2 \mathrm{~mm}$. Subjects with favorable response post-treatment were found to have a greater degree of overjet (OJ) compared to those with unfavorable responses at baseline $(\mathrm{p}=0$. 003).

\section{Discussion}

OSAS is a chronic disorder that often requires long life care. CPAP is a well-established treatment option for patients with severe OSAS, but it has been reported that $5 \%$ to $50 \%$ of these patients refuse CPAP or discontinue CPAP use within the first week. The equipment is relatively bulky, noisy, and demands electricity. Some patients complain of disturbing side effects, including feelings of inconvenience and discomfort, poor mask fit, claustrophobia, and nasal problems $[13,14]$.

Mandibular advancing appliances (MAA) became the predominant form of dental therapy for sleep disordered breathing in 1990's [15]. Although the American Sleep Disorders Association [16] has asserted that dental appliances can be considered when patients fail with CPAP treatment. In severe OSA, there is only scanty evidence available indicating that dental appliances are effective.

In our study, at the 6-month follow-up, the patients were highly satisfied with the effects of the dental appliance treatment with regard to daytime sleepiness, episodes of apnea, and snoring. Daytime sleepiness as measured by the ESS questionnaire, showed a significant decrease in the ESS score between baseline and the 6-month follow-up. The mean ESS score of $7.7 \pm 4.0$ after treatment in our study is comparable with the ESS score of 7.6 in the study by Johns [17]. Also, the mean ESS score in our patients at the 6-month follow-up was similar to the reported level in other studies $[18,19]$. These results suggest that the dental appliance had a clinically favorable effect on daytime sleepiness in patients with severe OSA. This finding is possibly attributed to two causes. The first one may be related to the fact that MAA used by those patients were constructed by advancing the mandible anteriorly. The second suggested cause is the simplicity and patient satisfaction with wearing their appliances. The first explanation may be related to the fact that MAA advances the mandible, which in turn advances the tongue passively, and the soft palate anteriorly. Eventually, this might result in enlarging the hypopharyngeal airway.

There is some evidence indicating that moderate to severe OSA is closely associated with obesity [20]. In the current study, we found that nonresponders after oral appliance treatment had a higher BMI than responders, a finding consistent with Millman [21].
Our selected subjects did not tolerate CPAP at the beginning and had expressed interest on alternative treatment options; those with dental or periodontal problems were excluded. Our subjects achieved good OA compliance and have reported some minor, side effects which were found to be acceptable and transient in most of the cases. Only a few of them did not tolerate OA after trying the device for a few nights.

Arousals and stage 1sleep increase in patients with obstructive sleep apnea on account of slow-wave sleep and rapid eye movement sleep $[22,23]$. In the present study, the mandibular advancement device reduced the obstructive apnea-hypopnea index and the arousal index and does not influence sleep in a negative way.

The present study has limitations. First, no control group was used. Second, the sample size might not have been large enough, absence of an objective method for measurement of compliance of oral appliance. A further study on a large scale of patients is required.

\section{Conclusion}

A significant improvement in AHI, minimum oxygen desaturation, and the arousal index time with the MAA, few side effects, better patient satisfaction, and compliance after 6 months of treatment are results indicating that oral appliance treatment does not influence sleep in a negative way and could be an alternative to CPAP for patients with severe OSA.

\section{References}

1. Sucena M1, Liistro G, Aubert G, Rodenstein DO, Pieters T (2006) Continuous positive airway pressure treatment for sleep apnoea: compliance increases with time in continuing users. Eur Respir $\mathrm{J}$ 27: 761-766.

2. Willis FB1, Isley AL, Geda YE, Shaygan A, Quarles L 4th, et al. (2008) Resolution of syncope with treatment of sleep apnea. J Am Board Fam Med 21: 466-468.

3. Gottlieb DJ1, Yenokyan G, Newman AB, O'Connor GT, Punjabi NM, et al (2010) Prospective study of obstructive sleep apnea and incident coronary heart disease and heart failure: the sleep heart health study. Circulation 122 352-360.

4. Pataka AD1, Frangulyan RR, Mackay TW, Douglas NJ, Riha RL (2012) Narcolepsy and sleep-disordered breathing. Eur J Neurol 19: 696-702.

5. Kim JH1, Kwon MS, Song HM, Lee BJ, Jang YJ, et al. (2009) Compliance with positive airway pressure treatment for obstructive sleep apnea. Clin Exp Otorhinolaryngol 2: 90-96.

6. Aloia MS1, Knoepke CE, Lee-Chiong T (2010) The new local coverage determination criteria for adherence to positive airway pressure treatment testing the limits? Chest 138: 875-879.

7. Hoffstein V1, Viner S, Mateika S, Conway J (1992) Treatment of obstructive sleep apnea with nasal continuous positive airway pressure. Patient compliance, perception of benefits, and side effects. Am Rev Respir Dis 145 841-845.

8. Rauscher H1, Formanek D, Popp W, Zwick H (1993) Self-reported vs measured compliance with nasal CPAP for obstructive sleep apnea. Chest 103: 16751680.

9. Gagnadoux F1, Fleury B, Vielle B, Pételle B, Meslier N, et al. (2009) Titrated mandibular advancement versus positive airway pressure for sleep apnoea. Eur Respir J 34: 914-920.

10. Marklund M1, Franklin KA, Sahlin C, Lundgren R (1998) The effect of a mandibular advancement device on apneas and sleep in patients with obstructive sleep apnea. Chest 113: 707-713.

11. Lindman R1, Bondemark $L$ (2001) A review of oral devices in the treatment of habitual snoring and obstructive sleep apnoea. Swed Dent J 25: 39-51.

12. Johns MW (1991) A new method for measuring daytime sleepiness: the Epworth sleepiness scale. Sleep 14: 540-545.

13. Chan AS1, Phillips CL, Cistulli PA (2010) Obstructive sleep apnoea--an update Intern Med J 40: 102-106. 
Citation: Yousef AM, El-Waseef F (2014) Can Oral Appliances be an Alternative Treatment for Severe Obstructive Sleep Apnea Syndrome Patients? J Pulm Respir Med 4: 182. doi:10.4172/2161-105X.1000182

14. Engleman HM, Wild MR. (2003) Improving CPAP use by patients with the sleep apnea/hypopnea syndrome. Sleep Med Rev. 7:81-99

15. Lowe AA (2000) The durability of intraoral devices for snoring and sleep apnea: another view. J Can Dent Assoc 66: 486-487.

16. [No authors listed] (1995) Practice parameters for the treatment of snoring and obstructive sleep apnea with oral appliances. American Sleep Disorders Association. Sleep 18: 511-513.

17. Johns MW (1992) Reliability and factor analysis of the Epworth Sleepiness Scale. Sleep 15: 376-381.

18. Kiely JL1, McNicholas WT (1997) Bed partners' assessment of nasa continuous positive airway pressure therapy in obstructive sleep apnea. Chest 111: $1261-1265$

19. Engleman HM1, Kingshott RN, Wraith PK, Mackay TW, Deary IJ, et al. (1999)
Randomized placebo-controlled crossover trial of continuous positive airway pressure for mild sleep Apnea/Hypopnea syndrome. Am J Respir Crit Care Med 159: 461-467.

20. Wittels EH1, Thompson S (1990) Obstructive sleep apnea and obesity. Otolaryngol Clin North Am 23: 751-760.

21. Millman RP1, Rosenberg CL, Carlisle CC, Kramer NR, Kahn DM, et al. (1998) The efficacy of oral appliances in the treatment of persistent sleep apnea after uvulopalatopharyngoplasty. Chest 113: 992-996.

22. Carskadon MA, Dement WC. (1994) Normal human sleep: an overview. In Kryger MH, Roth T, Dement WC, eds. Principles and practice of sleep medicine. 2nd ed. Philadelphia: WB Saunders. 16-25

23. Collard P1, Dury M, Delguste P, Aubert G, Rodenstein DO (1996) Movement arousals and sleep-related disordered breathing in adults. Am J Respir Crit Care Med 154: 454-459. 